\title{
Dynamic stabilization for L4-5 spondylolisthesis: comparison with minimally invasive transforaminal lumbar interbody fusion with more than 2 years of follow-up
}

\author{
Chao-Hung Kuo, MD, ,,2 Peng-Yuan Chang, MD,, Jau-Ching Wu, MD, PhD, 1,2 \\ Hsuan-Kan Chang, MD, ${ }^{1,2}$ Li-Yu Fay, MD, ${ }^{1-3}$ Tsung-Hsi Tu, MD, ${ }^{1,2,4}$ Henrich Cheng, MD, PhD, ${ }^{1-3}$ and \\ Wen-Cheng Huang, MD, $\mathrm{PhD}^{1,2}$
}

\begin{abstract}
${ }^{1}$ Department of Neurosurgery, Neurological Institute, Taipei Veterans General Hospital; ${ }^{2}$ School of Medicine and ${ }^{3}$ nnstitute of Pharmacology, National Yang-Ming University; and ${ }^{4}$ Molecular Medicine Program, Taiwan International Graduate Program, Academia Sinica, Taipei, Taiwan
\end{abstract}

\begin{abstract}
OBJECTIVE In the past decade, dynamic stabilization has been an emerging option of surgical treatment for lumbar spondylosis. However, the application of this dynamic construct for mild spondylolisthesis and its clinical outcomes remain uncertain. This study aimed to compare the outcomes of Dynesys dynamic stabilization (DDS) with minimally invasive transforaminal lumbar interbody fusion (MI-TLIF) for the management of single-level spondylolisthesis at L4-5.

METHODS This study retrospectively reviewed 91 consecutive patients with Meyerding Grade I spondylolisthesis at L4-5 who were managed with surgery. Patients were divided into 2 groups: DDS and MI-TLIF. The DDS group was composed of patients who underwent standard laminectomy and the DDS system. The MI-TLIF group was composed of patients who underwent MI-TLIF. Clinical outcomes were evaluated by visual analog scale for back and leg pain, Oswestry Disability Index, and Japanese Orthopaedic Association scores at each time point of evaluation. Evaluations included radiographs and CT scans for every patient for 2 years after surgery.
\end{abstract}

RESULTS A total of 86 patients with L4-5 spondylolisthesis completed the follow-up of more than 2 years and were included in the analysis (follow-up rate of $94.5 \%$ ). There were 64 patients in the DDS group and 22 patients in the MITLIF group, and the overall mean follow-up was 32.7 months. Between the 2 groups, there were no differences in demographic data (e.g., age, sex, and body mass index) or preoperative clinical evaluations (e.g., visual analog scale back and leg pain, Oswestry Disability Index, and Japanese Orthopaedic Association scores). The mean estimated blood loss of the MI-TLIF group was lower, whereas the operation time was longer compared with the DDS group (both $p<0.001$ ). For both groups, clinical outcomes were significantly improved at $6,12,18$, and 24 months after surgery compared with preoperative clinical status. Moreover, there were no differences between the 2 groups in clinical outcomes at each evaluation time point. Radiological evaluations were also similar and the complication rates were equally low in both groups.

CONCLUSIONS At 32.7 months postoperation, the clinical and radiological outcomes of DDS were similar to those of MI-TLIF for Grade I degenerative spondylolisthesis at L4-5. DDS might be an alternative to standard arthrodesis in mild lumbar spondylolisthesis. However, unlike fusion, dynamic implants have issues of wearing and loosening in the long term. Thus, the comparable results between the 2 groups in this study require longer follow-up to corroborate.

http://thejns.org/doi/abs/10.3171/2015.10.FOCUS15441

KEY WORDS Dynesys; TLIF; minimally invasive; spondylolisthesis; functional outcome

$\mathrm{R}$ ADICULOPATHY, back pain, and neurogenic claudication are common clinical presentations of lumbar degenerative spondylolisthesis. Treatment frequently begins with medication, local injection, and physical therapy. For those patients who are refractory to conserva- tive management, surgery has demonstrated substantially greater improvement in degenerative spondylolisthesis with stenosis. ${ }^{29,30}$ Surgical decompression of the neural elements obviously facilitates relief of neurological symptoms. Moreover, stabilization is also considered crucial in

ABBREVIATIONS ASD = adjacent-segment disease; DDS = Dynesys dynamic stabilization; EBL = estimated blood loss; JOA = Japanese Orthopaedic Association; MI = minimally invasive; ODI = Oswestry Disability Index; PET = polyethylene-terephthalate; TLIF = transforaminal lumbar interbody fusion; VAS = visual analog scale. SUBMITTED August 31, 2015. ACCEPTED October 8, 2015. INCLUDE WHEN CITING DOI: 10.3171/2015.10.FOCUS15441. 
such cases because it ameliorates the pathological motion at the index level. Although there is still inadequate evidence of the optimal surgical management method, interbody fusion may improve the rate of arthrodesis and can be performed via various surgical approaches (e.g., anterior, posterior, and transforaminal).., 26

The techniques applied for arthrodesis have evolved over the years. In the last several decades, the introduction of pedicle screws, interbody cages, and various types of biologics and grafting materials has increased the rate of success for spinal fusion. Symptomatic degenerative spondylolisthesis with spinal stenosis at L4-5 is one of the most frequent indications for lumbar interbody fusion..$^{14,20}$ Harms and Rolinger first described the surgical approach of transforaminal lumbar interbody fusion (TLIF) in 1982. ${ }^{9}$ Since then, the technique has been widely adapted and used worldwide. Furthermore, with technological improvements in recent years, the surgical approach of minimally invasive (MI)-TLIF has gained popularity and its effectiveness has been demonstrated for spondylolisthesis. ${ }^{16,18,23,27}$

In the recent decade, dynamic stabilization has been an emerging option for the management of lumbar spondylosis. Dynamic stabilization methods include interspinous devices and pedicle screw-based stabilization systems. The Dynesys dynamic stabilization (DDS; Zimmer Biomet) system is the subject of many published reports on its application for the management of lumbar degenerative disc disease, ${ }^{6,13,31,32}$ spondylolisthesis, ${ }^{7,8,10,20,22}$ and even degenerative scoliosis. ${ }^{3,4,15}$ However, the application of these dynamic constructs for spondylolisthesis and its clinical outcomes remain unclear. Therefore, this study aimed to compare the outcomes of DDS with those of standard lumbar fusion surgery via MI-TLIF.

\section{Methods \\ Patient Population}

Consecutive patients who underwent surgical management for single-level Meyerding Grade I spondylolisthesis at L4-5 between 2007 and 2012 were included. All clinical and radiological data of the 91 patients who underwent MI-TLIF or DDS were retrospectively reviewed. All patients had single-level (L4-5) lumbar degenerative disc disease or spinal stenosis with degenerative spondylolisthesis, causing symptoms such as neurogenic claudication, back pain, leg pain, or any combination of these. Every patient had a poor response to at least 12 weeks of conservative management, including medication and physical therapy, before proceeding to surgery. Patients were excluded for multiple levels of disc disease, Meyerding Grade II or higher spondylolisthesis, degenerative scoliosis, the presence of vertebral fracture, infection, tumor, or loss in follow-up. Patients with systemic connective tissue diseases, such as rheumatoid arthritis, ankylosing spondylitis, and psoriatic arthritis, were also excluded.

Every patient was evaluated preoperatively by MRI and anteroposterior and lateral dynamic radiographs for grading of spondylolisthesis. Patients were divided into 2 groups, DDS and MI-TLIF, for comparison of clinical and radiological outcomes. The choice between fusion (MI-
TLIF) or dynamic stabilization (DDS) was made by the patient and his/her family after discussion with the senior surgeon. Although the information was provided by the consultant with a balanced point of view, there may have been economic influences because DDS was approximately $50 \%$ less expensive than MI-TLIF and the patients had to pay.

Three attending surgeons (J.C.W., W.C.H., and H.C.) covered all patients (in both the DDS and MI-TLIF groups) and used similar surgical techniques taught by the senior author (H.C.).

\section{Surgical Techniques}

All patients underwent general anesthesia while in a prone position with adequate cushion. For DDS, standard total laminectomy of L-4 was performed via a posterior midline incision. Bilateral neural foramen and lateral recesses were decompressed by Kerrison rongeurs. Caution was taken to preserve the facet joints bilaterally. Generous subdermal dissection through the same midline skin incision allowed another 2 fascial incisions, 1 on each side, for dissection via the Wiltse approach. The Dynesys titanium alloy screws were then placed transpedicularly through the Wiltse plane without destruction of the facet joints. The constructs, polycarbonate-urethane spacers and polyethylene-terephthalate (PET) cords (Sulene-PET), were assembled. A tensioning instrument was used to exert tension on the PET cord with the specified preload. There was no intentional intraoperative reduction of any listhesis or change of the lordotic curvature. Lateral fluoroscopy was routinely used to ensure optimal positioning of the screws prior to the end of surgery.

For MI-TLIF, the primary decompression procedure and placement of the interbody cage were performed on the side that was more symptomatic. Tubular retractors, expandable or nonexpandable, were introduced under fluoroscopic guidance to the indexed facet joints. Laminotomy and facetectomy were then performed with a high-speed drill and Kerrison rongeur. The ligamentum flavum was then removed to expose the Kambin triangle. For decompression of the contralateral side, the tubular retractor was angled medially and the patient was tilted laterally. Extensive decompression was then performed, including decompression of central stenosis and the contralateral neural foramen. In selected cases, bilateral facetectomies were performed to ensure adequate decompression. After discectomy, local bone autografts and demineralized bone matrix were packed into a polyetheretherketone interbody cage and then inserted into the disc space along the trajectory of the working tube. After removal of the tubular retractor, placement of 4 pedicle screws percutaneously was done under fluoroscopy. All wounds were copiously irrigated before being closed in layers.

\section{Clinical and Radiographic Evaluations}

All medical records, operative notes, and radiological studies were retrospectively reviewed. Clinical outcomes were measured using the visual analog scale (VAS) for back and leg pain, the Oswestry Disability Index (ODI), and clinical symptom scores of the Japanese Orthopaedic 
Association (JOA). The patients completed questionnaires preoperatively and approximately $6,12,18$, and 24 months postoperatively. All clinical outcomes were evaluated by a special nurse assistant under the supervision of the physician during each clinic visit.

Preoperative and postoperative radiographs, including lateral flexion and extension views, were evaluated and compared. Postoperative CT was used to detect screw malpositioning or loosening. Screw loosening was defined by the presence of an initial halo sign (i.e., a radiolucent line around the implant $>1$-mm wide) followed by a double-halo sign on subsequent anteroposterior radiographs during follow-up. All patients had CT scans during followup for determination of possible screw loosening. ${ }^{1,13,21,32}$ All measurements were performed on a flat-panel screen of the picture archiving and communication system using quantitative analysis software. Radiographic interpretations were made by independent radiologists and neurosurgeons. To attain consensus in cases of ambiguity, CT scans and discussions were used for the final determination of a halo sign.

\section{Statistical Analysis}

Data are presented as the mean \pm SD for continuous variables and as frequency and percentages for categorical variables. All statistical tests were 2-tailed, and $\mathrm{p}<0.05$ was considered statistically significant by independent ttest, paired t-test, or chi-square test. All statistical analyses were performed using MedCalc software (Ostend).

\section{Results}

A total of 91 patients with L4-5 degenerative spondylolisthesis were included in the present study, and 86 patients completed the follow-up of more than 2 years (Table 1). Meyerding Grade I spondylolisthesis with stenosis was confirmed by lateral radiographs and MRI preoperatively. There were 64 patients (74\%) in the DDS group, who received standard open laminectomy and implantation of the Dynesys screws. There were 22 patients (26\%) in the MI-TLIF group, who underwent TLIF via tubular retractors and had percutaneous screw placement.

There were no statistically significant differences between the DDS and MI-TLIF groups in age, sex, and body mass index ( $\mathrm{p}=0.53,0.35$, and 0.36 , respectively). There were also no differences in patients' comorbidities, such as diabetes mellitus, hypertension, and cigarette smoking, between the 2 groups (Table 1).

The estimated blood loss (EBL) of the MI-TLIF group was significantly less than that of the DDS group (232.5 \pm 194.2 vs $348.0 \pm 242.2 \mathrm{ml}, \mathrm{p}<0.001$ ), whereas the mean operation time of the MI-TLIF group was longer than that of the DDS group $(4.5 \pm 1.3$ vs $3.3 \pm 1.0$ hours, $\mathrm{p}<0.001)$ (Table 2).

\section{VAS Back and Leg Pain Scores}

For patients in the DDS group, mean VAS scores of back and leg pain improved significantly after the operation (back pain from $6.4 \pm 3.0$ preoperatively to $2.3 \pm 2.3$ postoperatively; leg pain from $7.1 \pm 2.5$ to $2.3 \pm 2.4$; both $\mathrm{p}$ $<0.001)$. Similarly, for patients in the MI-TLIF group, the mean VAS scores improved significantly after the operation (back pain from $6.9 \pm 2.1$ preoperatively to $2.9 \pm 2.6$ postoperatively; leg pain from $6.0 \pm 2.9$ to $2.1 \pm 2.3$; both $\mathrm{p}<0.001)$. Furthermore, there were no differences in the VAS leg (Fig. 1) or back (Fig. 2) pain scores between the 2 groups at each time point of evaluation, including preoperatively and at postoperative follow-ups (Tables 1 and 2).

\section{ODI and JOA Scores}

Both the DDS and MI-TLIF groups had similar improvement in ODI scores after surgery. The ODI scores of the DDS group improved from $24.7 \pm 9.6$ preoperatively to $10.1 \pm 7.7$ postoperatively $(\mathrm{p}<0.001)$. The ODI score also improved significantly in the MI-TLIF group from $23.0 \pm$ 7.7 preoperatively to $9.9 \pm 7.1$ postoperatively $(p<0.001)$. Moreover, there were no inter- or intragroup differences at any time point of evaluation (Fig. 3, Tables 1 and 2).

Both the DDS and MI-TLIF groups had similar improvement in JOA scores after surgery. The JOA scores of the DDS group improved from $5.0 \pm 2.8$ preoperatively to $10.1 \pm 3.4$ postoperatively ( $\mathrm{p}<0.001)$. The JOA scores also improved significantly in the MI-TLIF group, from $5.9 \pm 2.9$ preoperatively to $10.1 \pm 3.4$ postoperatively $(\mathrm{p}$ $<0.001$ ). Furthermore, there were no inter- or intragroup differences at any time point of evaluation (Fig. 4, Tables 1 and 2).

\section{Adjacent-Segment Disease}

The incidences of adjacent-segment disease (ASD) were not significantly different between the DDS and MI-TLIF groups $(23.4 \%$ vs $31.8 \%, p=0.62)$. After a meticulous review of postoperative image evaluations, ${ }^{17} 22$ patients (15 and 7 patients from the DDS and MI-TLIF groups, respectively) were found to have radiological evidence of ASD (e.g., loss of disc height, disc degeneration/herniation, instability, listhesis, and hypertrophic facet arthritis) during the study period (Table 3). Although none of the patients were symptomatic or have had any clinical correlations to date, longer-term follow-up is warranted for these patients.

\section{Complications of Surgery}

In the DDS group, 1 patient had incidental durotomy during surgery, which was repaired without further complications. One patient in the DDS group underwent secondary surgery due to wound infection. Five patients had asymptomatic screw loosening, as determined by followup image evaluations. All 5 patients with loosened screws had minimal symptoms, and the halo signs remained the same without progression or further screw breakage. Thus, none of them required further surgery (Table 2).

In the MI-TLIF group, 1 patient had secondary surgery for screw revision due to malpositioning of a right L-5 pedicle screw. There were no neurological deficits in any of the patients who experienced complications.

\section{Illustrative Cases Case 1}

A 69-year-old man had intermittent low-back pain for more than 2 years. He presented with sciatica, numbness, and neurogenic claudication. An MRI study demonstrated 
TABLE 1. Comparison of demographic and clinical characteristics of 2 groups of patients who underwent DDS or MI-TLIF at the L4-5 level

\begin{tabular}{|c|c|c|c|}
\hline \multirow[b]{2}{*}{ Characteristic } & \multicolumn{2}{|c|}{ Group } & \multirow[b]{2}{*}{ p Value } \\
\hline & DDS & MI-TLIF & \\
\hline No. of pts & 64 & 22 & \\
\hline Age $(y r s)^{*}$ & $59.0 \pm 12.2$ & $57.2 \pm 11.6$ & 0.53 \\
\hline Sex (F/M) & $38: 26$ & $16: 6$ & 0.35 \\
\hline $\mathrm{BMI}^{*}$ & $24.4 \pm 2.8$ & $25.2 \pm 3.0$ & 0.36 \\
\hline Diabetes mellitus & $10 / 64$ & $4 / 22$ & 0.94 \\
\hline Hypertension & $19 / 64$ & $8 / 22$ & 0.81 \\
\hline Cigarette smoking & $5 / 64$ & $5 / 22$ & 0.94 \\
\hline \multicolumn{4}{|l|}{ Preop symptoms } \\
\hline Neurogenic claudication & $35 / 64$ & $12 / 22$ & 0.41 \\
\hline Radicular pain & $64 / 64$ & $22 / 22$ & \\
\hline \multicolumn{4}{|l|}{ Spondylolisthesis type } \\
\hline Spondylotic & $0 / 64$ & $2 / 22$ & \\
\hline Degenerative & $64 / 64$ & $20 / 22$ & \\
\hline \multicolumn{4}{|l|}{ Preop scores* } \\
\hline VAS back pain & $6.4 \pm 3.0$ & $6.9 \pm 2.1$ & 0.46 \\
\hline VAS leg pain & $7.1 \pm 2.5$ & $6.0 \pm 2.9$ & 0.11 \\
\hline ODI & $24.7 \pm 9.6$ & $23.0 \pm 7.7$ & 0.42 \\
\hline JOA & $5.0 \pm 2.8$ & $5.9 \pm 2.9$ & 0.33 \\
\hline
\end{tabular}

$\mathrm{BMI}=$ body mass index; pts $=$ patients.

* Values are presented as the mean \pm SD.

spinal stenosis at L4-5 caused by the bulging of anular fibrosus and hypertrophic ligamentum flavum (Fig. 5A) and Grade I degenerative spondylolisthesis (Fig. 5C). Due to poor response to medical management and physical ther- apy, surgical decompression and stabilization were suggested. Standard midline total laminectomy of L-4 with DDS implantation were performed. The patient's symptoms remarkably improved after the surgery and his post-

TABLE 2. Comparison of perioperative clinical characteristics and complications of 2 groups of patients who underwent DDS or MI-TLIF at the L4-5 level

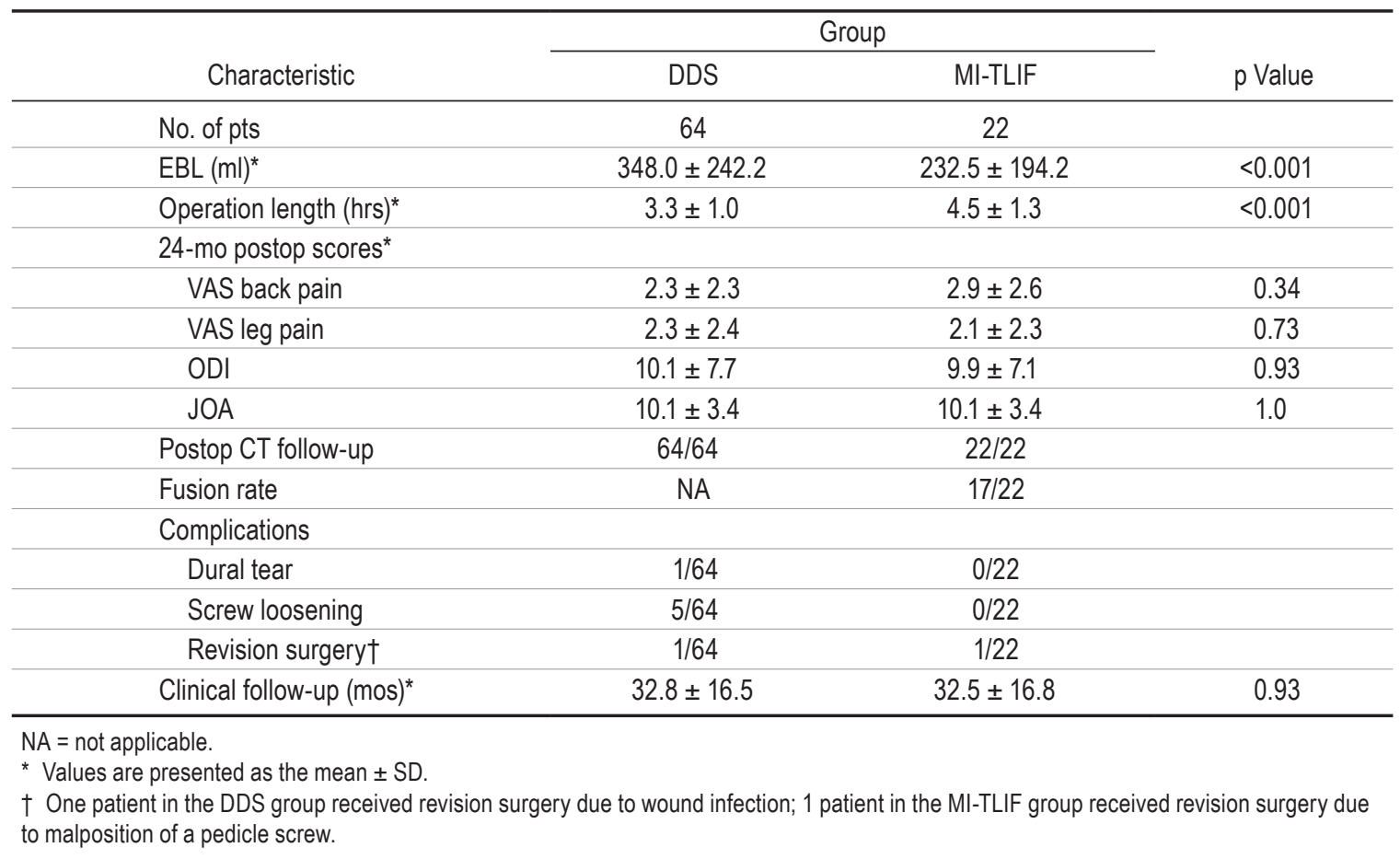




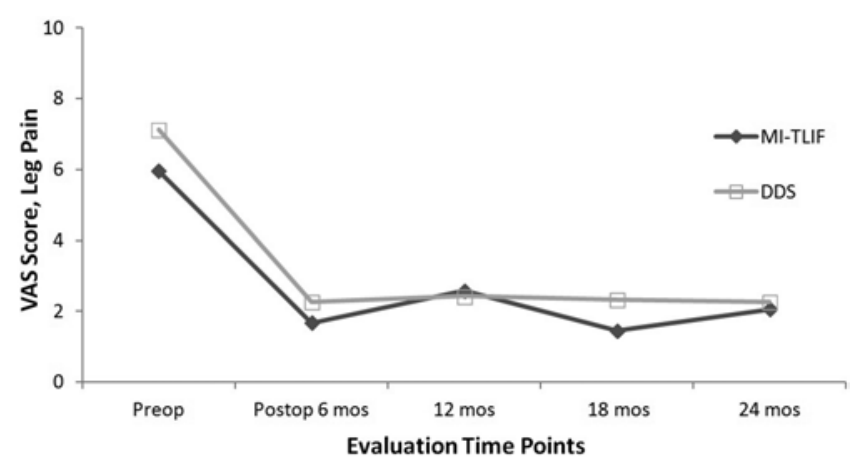

FIG. 1. Clinical outcomes measured by average VAS score for leg pain at different time points suggest significant improvement postoperatively. There was no significant difference between patients who underwent MI-TLIF versus DDS.

operative course was uneventful. The 2-year follow-up MR image showed the stable construct of DDS (Figs. 5B and $\mathrm{D}$ and $7 \mathrm{~A}$ and $\mathrm{B})$.

\section{Case 2}

A 64-year-old woman had low-back pain with radiating pain down to her bilateral thighs for approximately 3 months, despite physical therapy. Also, there had been progressive neurogenic claudication for several months. Her MRI demonstrated spinal stenosis due to the bulging of anular fibrosus at L4-5 and hypertrophy of ligamentum flavum (Fig. 6A). Lateral radiographs revealed Meyerding Grade I spondylolisthesis at L4-5 (Fig. 6C). MI-TLIF was performed without adverse events. The patient's symptoms improved greatly after surgery and her postoperative course was uneventful for up to 2 years of follow-up (Figs. $6 \mathrm{~B}$ and $\mathrm{D}$ and $7 \mathrm{C}$ and $\mathrm{D}$ ).

\section{Discussion}

To our knowledge, the present study is the first to compare DDS with MI-TLIF for the management of Meyerding Grade I spondylolisthesis, specifically at L4-5. All patients had single-level spondylolisthesis and presented with symptoms of stenosis and instability. Patients were placed into the DDS and MI-TLIF groups (64 and 22 pa-

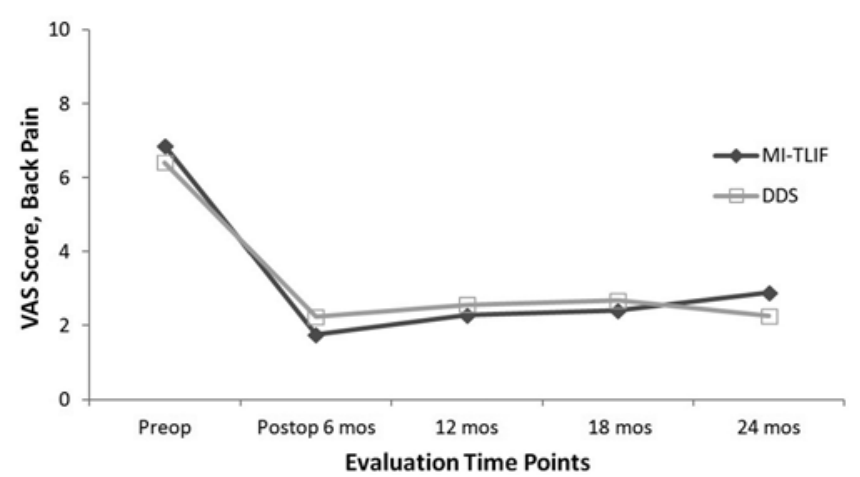

FIG. 2. Clinical outcomes measured by average VAS score for back pain at different time points suggest significant improvement postoperatively. There was no significant difference between patients who underwent MI-TLIF versus DDS.

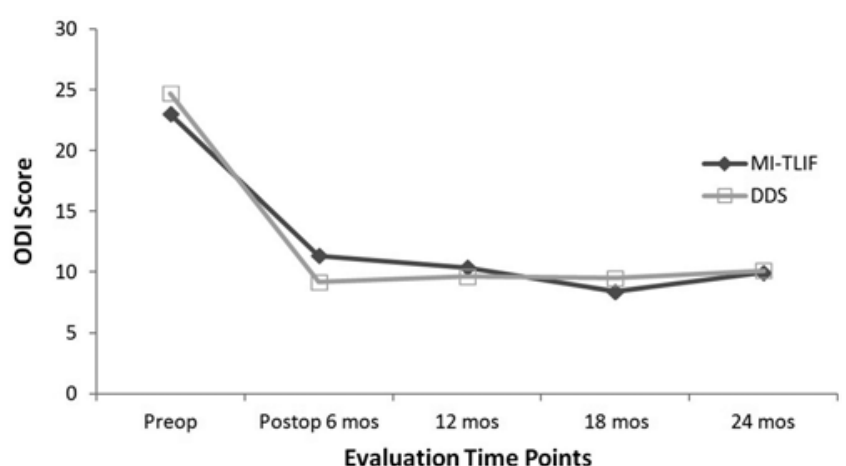

FIG. 3. Clinical outcomes measured by average ODI score at different time points suggest significant improvement postoperatively. There was no significant difference between patients who underwent MI-TLIF versus DDS.

tients, respectively). Clinical (including VAS back and leg pain, ODI, and JOA scores) and radiological outcomes were compared. The follow-up period was a minimum of 2 years (mean 32.7 months). There were few differences between patients who received DDS versus MI-TLIF surgery. The present series of 86 patients demonstrated high rates of neurological improvement with low rates of adverse events. Both DDS and MI-TLIF yielded satisfactory clinical and radiological outcomes. Therefore, DDS appeared to be an acceptable alternative to MI-TLIF for the management of mild spondylolisthesis at L4-5.

Standard open TLIF has been an accepted surgical option for patients with lumbar spondylolisthesis..$^{11}$ During the last decade, MI-TLIF has been an emerging option. A number of series have demonstrated that MI-TLIF provided similar clinical and functional outcomes with less soft-tissue damage, decreased blood loss, reduced length of hospital stay, and earlier recovery than standard open TLIF. $^{11,12,28}$ In a retrospective study of 100 patients with low-grade spondylolisthesis, there were no statistical differences between 2 groups of patients who underwent open versus minimally invasive approaches with more than 4 years of follow-up. The clinical and radiological outcomes were similar, although longer operative time and more EBL were demonstrated in the group of patients who underwent open TLIF. ${ }^{33}$ Another retrospective study reviewed 42 patients with low-grade spondylolisthesis and equally

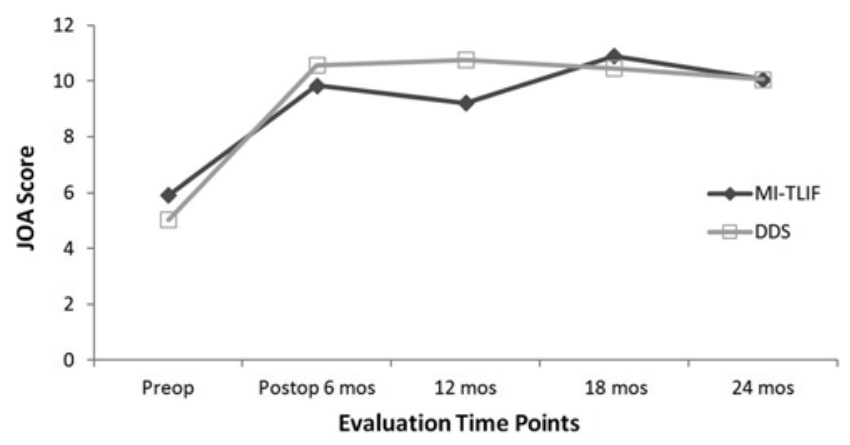

FIG. 4. Clinical outcomes measured by average clinical symptom JOA score at different time points suggest significant improvement postoperatively. There was no significant difference between patients who underwent MI-TLIF versus DDS. 
TABLE 3. Incidence of ASD in 86 patients with Meyerding Grade I spondylolisthesis at L4-5

\begin{tabular}{lrrcc}
\hline \multirow{2}{*}{ Group } & \multicolumn{2}{c}{ ASD } & & \\
\cline { 2 - 3 } & Yes* $^{*}$ & No & \% Incidence & p Value \\
\hline Total no. of pts & 22 & 64 & & \\
\hline DDS & 15 & 49 & 23.4 & \multirow{2}{*}{0.62} \\
\hline MI-TLIF & 7 & 15 & 31.8 & \\
\hline
\end{tabular}

* All 22 patients with ASD remained asymptomatic and did not require secondary surgery at the last follow-up.

divided the patients into MI- and open-TLIF groups. They demonstrated more EBL and longer hospital stay in the open group. ${ }^{2}$ Parker et al. retrospectively compared $100 \mathrm{pa}-$ tients with Grade I lumbar degenerative spondylolisthesis treated with either MI-TLIF or open TLIF (50 patients in each group). ${ }^{19}$ There were no differences in functional outcomes, including VAS back and leg pain and ODI scores, between the groups. However, time of return to work and length of hospital stay were significantly shorter in the MITLIF group than the open-TLIF group.

There are a number of published series that used DDS to treat symptomatic spondylolisthesis. Hoppe et al. reported 39 consecutive patients with degenerative lumbar spondylolisthesis at L4-5 treated with laminectomy and DDS. During a mean follow-up of 7.2 years, they demonstrated that over $80 \%$ of patients' back and leg pain improved significantly following surgery..$^{10}$ Payer et al. reported another retrospective study, which included $30 \mathrm{pa}-$ tients with single-level degenerative spondylolisthesis and spinal stenosis who were followed up for 2 years. Payer and colleagues demonstrated improved functional outcomes including VAS scores of leg and back pain, which improved significantly. Also, the average pain-free walking distance improved from $500 \mathrm{~m}$ preoperatively to more than $2000 \mathrm{~m}$ at 2 years after surgery. ${ }^{20} \mathrm{~A}$ prospective study by Schnake et al. included 26 patients who underwent decompression and DDS for lumbar spinal stenosis and degenerative spondylolisthesis. Schnake and colleagues also demonstrated a significant decrease in leg pain and improvement in walking distance after DDS. Thus, the authors stated that DDS not only improved clinical and functional outcomes but also restored normal function of the spinal segments while protecting the adjacent segments. ${ }^{24}$ Fay et al. reported on 72 patients managed with 1- or 2-level DDS, and they demonstrated intervertebral disc rehydration in younger patients, with significant improvement of clinical outcomes. ${ }^{6}$

Unlike spinal fusion surgery, there has always been the concern of screw loosening in patients managed with dynamic stabilization. Instrumentation failure, including loosened and broken screws, remains and will be the most worrisome issue of DDS surgery, although few side effects have been reported. In a large series of DDS surgeries, a total of 658 screws in 126 patients were followed up for 37 months; $4.7 \%$ of screws became loose..$^{32}$ These authors also stated that at least 1 screw became loose in $19.8 \%$ of patients, and older patients or those with diabetes had a higher risk of screw loosening. Furthermore, those patients who had screw loosening were asymptomatic and had very similar clinical outcomes to those patients who had not experienced screw loosening. Ko at el. published a study of 71 patients who underwent 1- or 2-level DDS, and reported screw loosening in $19.7 \%$ of patients involving $4.6 \%$ of screws.$^{13}$ These authors also found few symptoms related to screw loosening. Others have reported rates of screw loosening ranging from $6.7 \%$ to $18 \%$ in various series using different methods of assessment. ${ }^{20,25}$ Interestingly, there were very few reports that addressed adverse events or secondary surgery related to loosened screws. Most of the patients who underwent DDS surgery had improved clinical symptoms, including those patients who had loosened screws.

There are limitations to the current study. This was a retrospective nonrandomized cohort. The choice of DDS or MI-TLIF was arbitrarily decided by the patient after
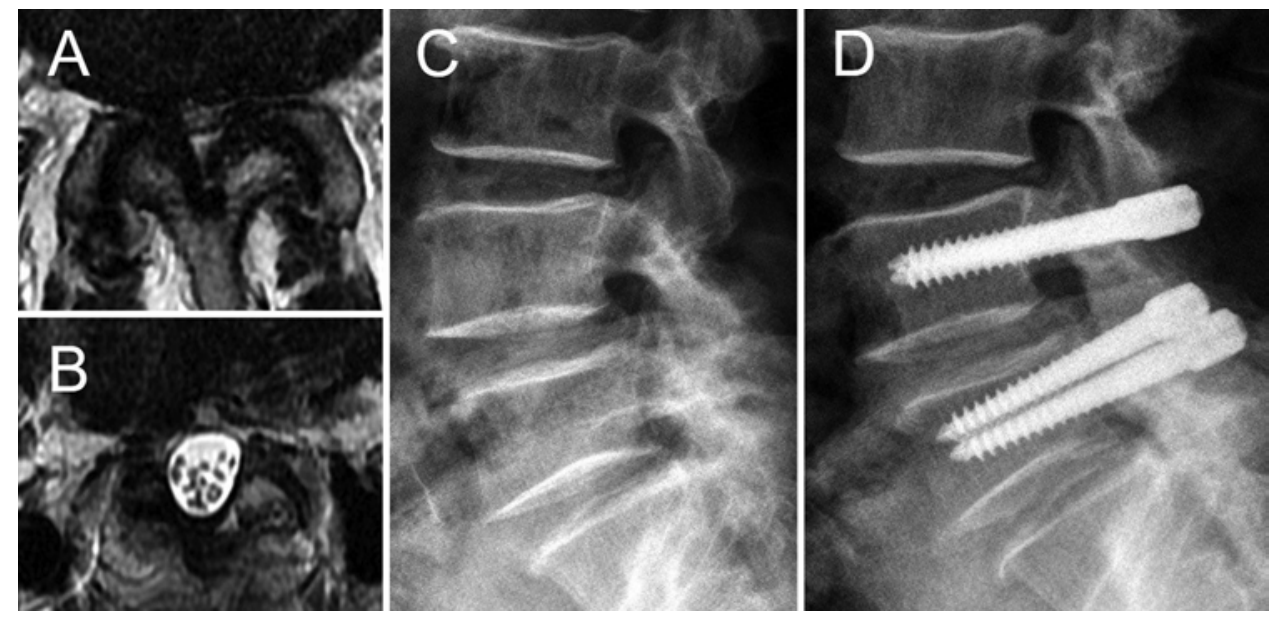

FIG. 5. Images obtained in the case of a 69 -year-old man with symptomatic spondylolisthesis. The preoperative MR image showed spinal stenosis at L4-5 caused by a bulging of anular fibrosus with hypertrophic ligamentum flavum (A), which was decompressed by total laminectomy (B). The preoperative lateral radiographs revealed Grade I degenerative spondylolisthesis (C). The 2-year follow-up radiograph demonstrated the stable construct of DDS (D). 

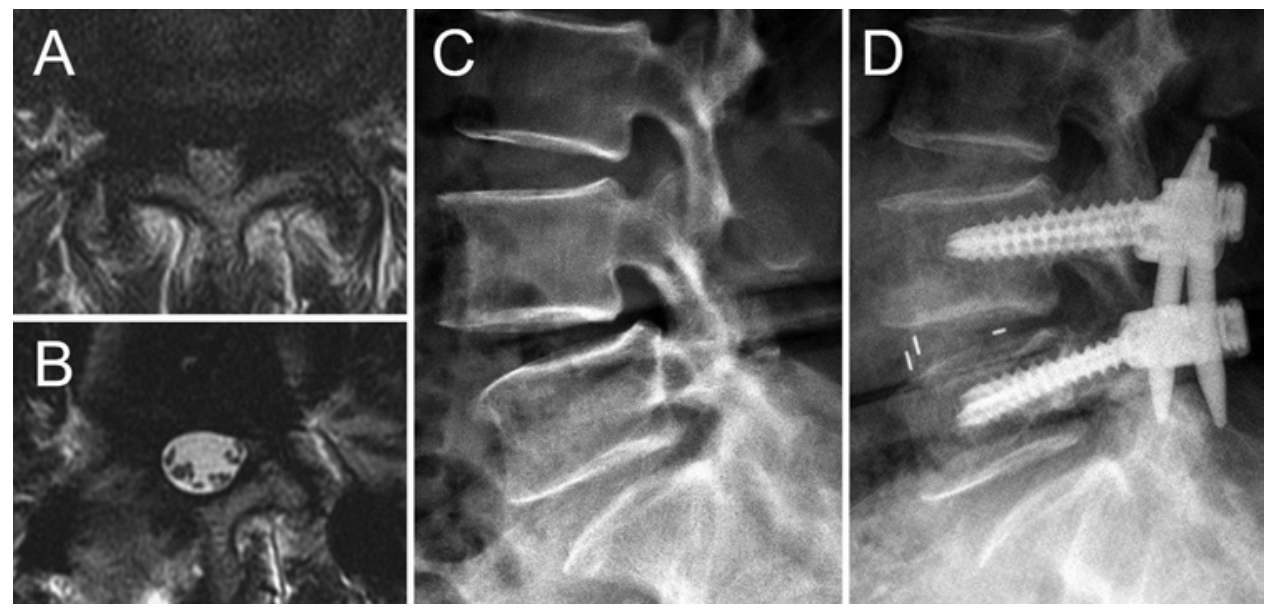

FIG. 6. Images obtained in the case of a 64-year-old woman who had been having low-back pain with radiating pain and progressive neurogenic claudication for several months. The preoperative MR image showed spinal stenosis due to the bulging of anular fibrosus at L4-5 and hypertrophy of ligamentum flavum (A), which was decompressed by MI-TLIF (B). The lateral radiographs demonstrated Meyerding Grade I spondylolisthesis at L4-5 (C). The postoperative course was still uneventful at the 2-year followup (D).

consultation with the senior surgeon. Although both options were clearly presented and well explained, the patients' choices could have been biased because the cost of MI-TLIF at the authors' institution was approximately twice as much as DDS. This might also account for more patients in the DDS group, although the expectations and psychological influences caused by the differences in price remain unclear. However, the series included consecutive patients with spondylolisthesis only at L4 -5 . This relatively uniform inclusion, substantiated by the similar preoperative conditions of both groups (Table 1), minimized confounding factors. There have been few studies in the literature limiting the pathology to only L4-5 and comparing DDS with MI-TLIF. Furthermore, the mean followup of more than 2.5 years is reasonable for spinal arthrodesis operations, but might be a bit short for DDS surgeries. The durability of the dynamic instrumentation remained uncertain, because screw loosening or progression of pathology needed a longer time for evaluation.

The optimal surgical management of degenerative spondylolisthesis is debated among spinal surgery experts. Although the present study demonstrated few differences between DDS and MI-TLIF, it did shed some light upon choice of procedure and future investigations. For younger patients with preserved disc height, minimal facet arthropathy, fewer radicular symptoms, and good bone quality, the application of DDS might be reasonably contemplated because it preserves motion and avoids interbody procedures. Nonetheless, for elderly patients who have osteopenia, severe spondylosis and arthropathy, incompetent facets, or advanced degenerative disc disease, it is safer to use spinal fusion procedures such as MI-TLIF. Because DDS uses less aggressive bony decompression than MI-TLIF and inevitably has a higher risk of screw loosening in the long term, it is better suited for patients with less degeneration. Future investigations are necessary to ascertain the durability of DDS, as well as its ability to halt lumbar degeneration.
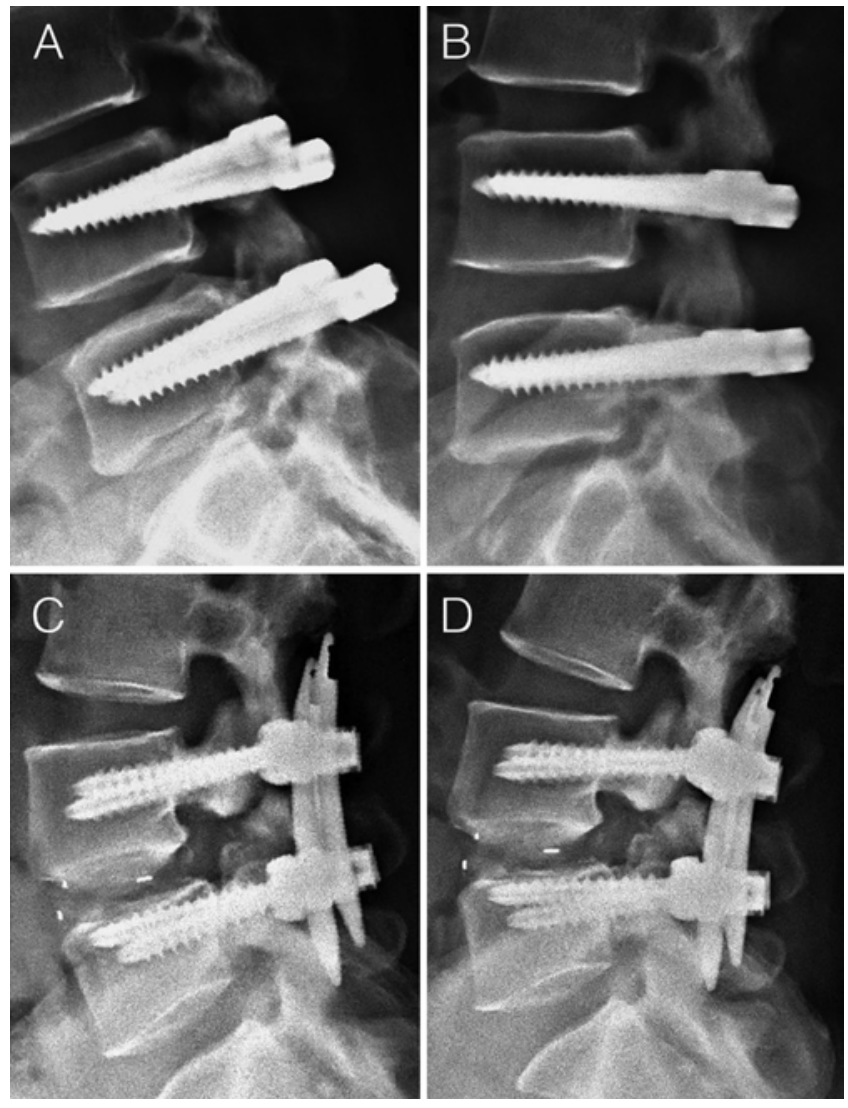

FIG. 7. The lateral flexion-extension radiographs show a 2-year followup image for each group of DDS (A and B) and MI-TLIF (C and D). These compare the preserved range of motion in the DDS group ( $A$ : flexion; $B$ : extension) and the limited range of motion with fusion in the MI-TLIF group (C: flexion; D: extension). 


\section{Conclusions}

At 32.7 months of follow-up, the clinical and radiological outcomes of DDS were similar to those of MI-TLIF for Grade I degenerative spondylolisthesis at L4-5. DDS may be an alternative to standard arthrodesis in mild lumbar spondylolisthesis. However, unlike fusion, dynamic implants have the issues of wearing and loosening in the long term. Thus, the comparable results between the 2 groups in this study require longer follow-up to corroborate.

\section{References}

1. Dakhil-Jerew F, Jadeja H, Cohen A, Shepperd JA: Interobserver reliability of detecting Dynesys pedicle screw using plain X-rays: a study on 50 post-operative patients. Eur Spine J 18: 1486-1493, 2009

2. Dhall SS, Wang MY, Mummaneni PV: Clinical and radiographic comparison of mini-open transforaminal lumbar interbody fusion with open transforaminal lumbar interbody fusion in 42 patients with long-term follow-up. J Neurosurg Spine 9:560-565, 2008

3. Di Silvestre M, Lolli F, Bakaloudis G: Degenerative lumbar scoliosis in elderly patients: dynamic stabilization without fusion versus posterior instrumented fusion. Spine J 14:1-10, 2014

4. Di Silvestre M, Lolli F, Bakaloudis G, Parisini P: Dynamic stabilization for degenerative lumbar scoliosis in elderly patients. Spine (Phila Pa 1976) 35:227-234, 2010

5. Eismont FJ, Norton RP, Hirsch BP: Surgical management of lumbar degenerative spondylolisthesis. J Am Acad Orthop Surg 22:203-213, 2014

6. Fay LY, Wu JC, Tsai TY, Tu TH, Wu CL, Huang WC, et al: Intervertebral disc rehydration after lumbar dynamic stabilization: magnetic resonance image evaluation with a mean followup of four years. Adv Orthop 2013:437570, 2013

7. Fay LY, Wu JC, Tsai TY, Wu CL, Huang WC, Cheng H: Dynamic stabilization for degenerative spondylolisthesis: evaluation of radiographic and clinical outcomes. Clin Neurol Neurosurg 115:535-541, 2013

8. Fayyazi AH, Ordway NR, Park SA, Fredrickson BE, Yonemura K, Yuan HA: Radiostereometric analysis of postoperative motion after application of Dynesys dynamic posterior stabilization system for treatment of degenerative spondylolisthesis. J Spinal Disord Tech 23:236-241, 2010

9. Harms J, Rolinger H: [A one-stager procedure in operative treatment of spondylolistheses: dorsal traction-reposition and anterior fusion (author's transl).] Z Orthop Ihre Grenzgeb 120:343-347, 1982 (Ger)

10. Hoppe S, Schwarzenbach O, Aghayev E, Bonel H, Berlemann U: Long-term outcome after monosegmental 14/5 stabilization for degenerative spondylolisthesis with the Dynesys device. J Spinal Disord Tech [epub ahead of print], 2012

11. Jhala A, Singh D, Mistry M: Minimally invasive transforaminal lumbar interbody fusion: Results of 23 consecutive cases. Indian J Orthop 48:562-567, 2014

12. Kaloostian PE, Gokaslan ZL: Evidence-based review of transforaminal lumbar interbody fusion: is minimally invasive better? World Neurosurg 82:65-67, 2014

13. Ko CC, Tsai HW, Huang WC, Wu JC, Chen YC, Shih YH, et al: Screw loosening in the Dynesys stabilization system: radiographic evidence and effect on outcomes. Neurosurg Focus 28(6):E10, 2010

14. Lawhorne TW III, Girardi FP, Mina CA, Pappou I, Cammisa FP Jr: Treatment of degenerative spondylolisthesis: potential impact of dynamic stabilization based on imaging analysis. Eur Spine J 18:815-822, 2009
15. Lee SE, Jahng TA, Kim HJ: Decompression and nonfusion dynamic stabilization for spinal stenosis with degenerative lumbar scoliosis: Clinical article. J Neurosurg Spine 21:585-594, 2014

16. McGirt MJ, Parker SL, Lerner J, Engelhart L, Knight T, Wang MY: Comparative analysis of perioperative surgical site infection after minimally invasive versus open posterior/ transforaminal lumbar interbody fusion: analysis of hospital billing and discharge data from 5170 patients. J Neurosurg Spine 14:771-778, 2011

17. Park P, Garton HJ, Gala VC, Hoff JT, McGillicuddy JE: Adjacent segment disease after lumbar or lumbosacral fusion: review of the literature. Spine (Phila Pa 1976) 29:19381944, 2004

18. Parker SL, Adogwa O, Witham TF, Aaronson OS, Cheng J, McGirt MJ: Post-operative infection after minimally invasive versus open transforaminal lumbar interbody fusion (TLIF): literature review and cost analysis. Minim Invasive Neurosurg 54:33-37, 2011

19. Parker SL, Mendenhall SK, Shau DN, Zuckerman SL, Godil SS, Cheng JS, et al: Minimally invasive versus open transforaminal lumbar interbody fusion for degenerative spondylolisthesis: comparative effectiveness and cost-utility analysis. World Neurosurg 82:230-238, 2014

20. Payer M, Smoll NR, Oezkan N, Tessitore E: Dynamic transpedicular stabilisation and decompression in single-level degenerative anterolisthesis and stenosis. Acta Neurochir (Wien) 156:221-227, 2014 [Erratum in Acta Neurochir (Wien) 156:759, 2014]

21. Sandén B, Olerud C, Petrén-Mallmin M, Johansson C, Larsson $\mathrm{S}$ : The significance of radiolucent zones surrounding pedicle screws. Definition of screw loosening in spinal instrumentation. J Bone Joint Surg Br 86:457-461, 2004

22. Schaeren S, Broger I, Jeanneret B: Minimum four-year follow-up of spinal stenosis with degenerative spondylolisthesis treated with decompression and dynamic stabilization. Spine (Phila Pa 1976) 33:E636-E642, 2008

23. Schizas C, Tzinieris N, Tsiridis E, Kosmopoulos V: Minimally invasive versus open transforaminal lumbar interbody fusion: evaluating initial experience. Int Orthop 33:16831688,2009

24. Schnake KJ, Schaeren S, Jeanneret B: Dynamic stabilization in addition to decompression for lumbar spinal stenosis with degenerative spondylolisthesis. Spine (Phila Pa 1976) 31:442-449, 2006

25. Segura-Trepichio M, Ferrández-Sempere D, López-Prats F, Segura-Ibáñez J, Maciá-Soler L: [Pedicular dynamic stabilization system. Functional outcomes and implant-related complications for the treatment of degenerative lumbar disc disease with a minimum follow-up of 4 years.] Rev Esp Cir Ortop Traumatol 58:85-91, 2014 (Span)

26. Sengupta DK, Herkowitz HN: Degenerative spondylolisthesis: review of current trends and controversies. Spine (Phila Pa 1976) 30 (6 Suppl):S71-S81, 2005

27. Sulaiman WA, Singh M: Minimally invasive versus open transforaminal lumbar interbody fusion for degenerative spondylolisthesis grades 1-2: patient-reported clinical outcomes and cost-utility analysis. Ochsner J 14:32-37, 2014

28. Wang J, Zhou Y: Perioperative complications related to minimally invasive transforaminal lumbar fusion: evaluation of 204 operations on lumbar instability at single center. Spine J 14:2078-2084, 2014

29. Weinstein JN, Lurie JD, Tosteson TD, Hanscom B, Tosteson AN, Blood EA, et al: Surgical versus nonsurgical treatment for lumbar degenerative spondylolisthesis. N Engl J Med 356:2257-2270, 2007

30. Weinstein JN, Lurie JD, Tosteson TD, Zhao W, Blood EA, Tosteson AN, et al: Surgical compared with nonoperative treatment for lumbar degenerative spondylolisthesis. Four- 
year results in the Spine Patient Outcomes Research Trial (SPORT) randomized and observational cohorts. J Bone Joint Surg Am 91:1295-1304, 2009

31. Welch WC, Cheng BC, Awad TE, Davis R, Maxwell JH, Delamarter R, et al: Clinical outcomes of the Dynesys dynamic neutralization system: 1-year preliminary results. Neurosurg Focus 22(1):E8, 2007

32. Wu JC, Huang WC, Tsai HW, Ko CC, Wu CL, Tu TH, et al: Pedicle screw loosening in dynamic stabilization: incidence, risk, and outcome in 126 patients. Neurosurg Focus 31(4):E9, 2011

33. Zairi F, Arikat A, Allaoui M, Assaker R: Transforaminal lumbar interbody fusion: comparison between open and mini-open approaches with two years follow-up. J Neurol Surg A Cent Eur Neurosurg 74:131-135, 2013

\section{Disclosures}

The authors report no conflict of interest concerning the materi- als or methods used in this study or the findings specified in this paper.

\section{Author Contributions}

Conception and design: Kuo, PY Chang. Acquisition of data: Kuo. Analysis and interpretation of data: Kuo. Drafting the article: Kuo, PY Chang, Fay. Critically revising the article: Fay. Reviewed submitted version of manuscript: Wu. Approved the final version of the manuscript on behalf of all authors: $\mathrm{Wu}$. Statistical analysis: Tu. Administrative/technical/material support: HK Chang, Tu, Cheng, Huang. Study supervision: Cheng, Huang.

\section{Correspondence}

Jau-Ching Wu, Department of Neurosurgery, Neurological Institute, Taipei Veterans General Hospital, Rm. 508, 17F, No. 201, Shih-Pai Rd., Sec. 2, Beitou, Taipei 11217, Taiwan. email: jauching@gmail.com. 